\title{
A copper nanocluster incorporated nanogel: Confinement-assisted emission enhancement for zinc ion detection in living cells
}

\author{
Xueqing Gao ${ }^{\mathrm{a}}$, Xuming Zhuang ${ }^{\mathrm{a}, \mathrm{b}, * *}$, Chunyuan Tian ${ }^{\mathrm{a}}$, Huitao Liu ${ }^{\mathrm{a}}$, Wing-Fu Lai ${ }^{\mathrm{c}}$, \\ Zhenguang Wang ${ }^{\mathrm{d}}$, Xuming Yang ${ }^{\mathrm{b}}$, Lingxin Chen ${ }^{\mathrm{e}, *}$, Andrey L. Rogach ${ }^{\mathrm{b}, \mathrm{f}}$ \\ ${ }^{a}$ College of Chemistry and Chemical Engineering, Yantai University, Yantai 264005, China \\ ${ }^{\mathrm{b}}$ Department of Materials Science and Engineering, and Centre for Functional Photonics (CFP), City University of Hong Kong, 83 Tat Chee Avenue, Kowloon, Hong Kong \\ Special Administrative Region \\ ${ }^{\mathrm{c}}$ Department of Applied Biology and Chemical Technology, Hong Kong Polytechnic University, Hong Kong Special Administrative Region \\ ${ }^{\mathrm{d}}$ College of Chemistry and Environmental Science, Hebei University, Baoding 071002, China \\ e Shandong Key Laboratory of Coastal Environmental Processes, CAS Key Laboratory of Coastal Environmental Processes and Ecological Remediation, Yantai Institute of \\ Coastal Zone Research, Chinese Academy of Sciences, Yantai 264003, China \\ ${ }^{\mathrm{f}}$ Shenzhen Research Institute, City University of Hong Kong, Shenzhen 518057, China
}

\section{A R T I C L E I N F O}

\section{Keywords:}

Copper nanoclusters

Glycol chitosan

Aggregation induced emission

Zinc(II) detection

Cell imaging

\begin{abstract}
A B S T R A C T
In this work, copper nanoclusters (CuNCs), in which cysteine served as both a reducing agent and a protection ligand, were synthesized, and impregnated into a nanogel of glycol chitosan (GC), forming a CuNCs@GC nanocomposite. The stability of CuNCs incorporated into a nanogel was significantly improved, while their photoluminescence (PL) intensity of CuNCs@GC could be tuned by controlling the pH of the system. The nanocomposite was used as a platform for the "turn on" detection of $\mathrm{Zn}^{2+}$, making use of the effect of aggregation induced emission (AIE) enhancement. By adjusting the pH of the system to 7.4, the PL of CuNCs@GC was turned down so as to minimize the background signal. In the presence of $\mathrm{Zn}^{2+}$, the nanoclusters in CuNCs@GC changed from the dispersed state to the aggregated state, which resulted in an increase of the PL intensity. A linear response between the PL intensity and the concentration of $\mathrm{Zn}^{2+}$, in the range from $1.5 \mu \mathrm{M}$ to $750 \mu \mathrm{M}$, was obtained, achieving a detection limit of $1.0 \mu \mathrm{M}$. Furthermore, the CuNCs@GC nanocomposite was found to be applicable to live cell imaging, owing to the high biocompatibility and protective effects of GC.
\end{abstract}

\section{Introduction}

Zinc ions $\left(\mathrm{Zn}^{2+}\right)$ are one of the trace elements that are essential to human health. They have been implicated in several biological processes, from growth and development to neurotransmission. The National Research Council of the US National Academy of Sciences has enacted a dietary guideline for zinc intake, since a deficiency of $\mathrm{Zn}^{2+}$ may hamper immune functions, retard the wound healing process, and delay growth and development [1,2]. Moreover, maintaining the normal level of $\mathrm{Zn}^{2+}$ can help to sustain proper functions of the brain and is therapeutic to patents showing depressive symptoms [3,4]. Therefore, it is important to establish a rapid, sensitive and selective method for the detection of $\mathrm{Zn}^{2+}$. Some traditional methods, including flame atomic absorption spectrometry [5], inductively coupled plasma atomic emission spectroscopy [6], ultraviolet-visible (UV-vis) spectrophotometry [7], high performance liquid chromatography [8], electrochemical sensors [9], and atomic absorption spectroscopy [10], have been used to detect $\mathrm{Zn}^{2+}$, but they often require tedious sample pretreatment and a series of expensive instruments. Importantly, many of these methods cannot be directly applied to detect $\mathrm{Zn}^{2+}$ in living cells. Photoluminescence (PL) based methods provide a promising alternative because of their high sensitivity, simplicity, high selectivity, and short detection time. As far as a typical PL detection method is concerned, the most important part is the luminescent probe, which should ideally be highly biocompatible, offer high PL quantum yield (QY), and low background signals [11-13].

Metal nanoclusters have recently emerged as luminescent materials, which not only are small in size and photostable but also possess a large

\footnotetext{
* Corresponding author.

** Corresponding author at: College of Chemistry and Chemical Engineering, Yantai University, Yantai 264005, China.

E-mail addresses: xqgao_ytu@163.com (X. Gao), xmzhuang@iccas.ac.cn (X. Zhuang), tianchunyuan_2005@163.com (C. Tian), liuht-ytu@163.com (H. Liu), rori0610@graduate.hku.hk (W.-F. Lai),wzg583@163.com (Z. Wang),xuminyang2-c@my.cityu.edu.hk (X. Yang), 1xchen@yic.ac.cn (L. Chen), andrey.rogach@cityu.edu.hk (A.L. Rogach).
} 


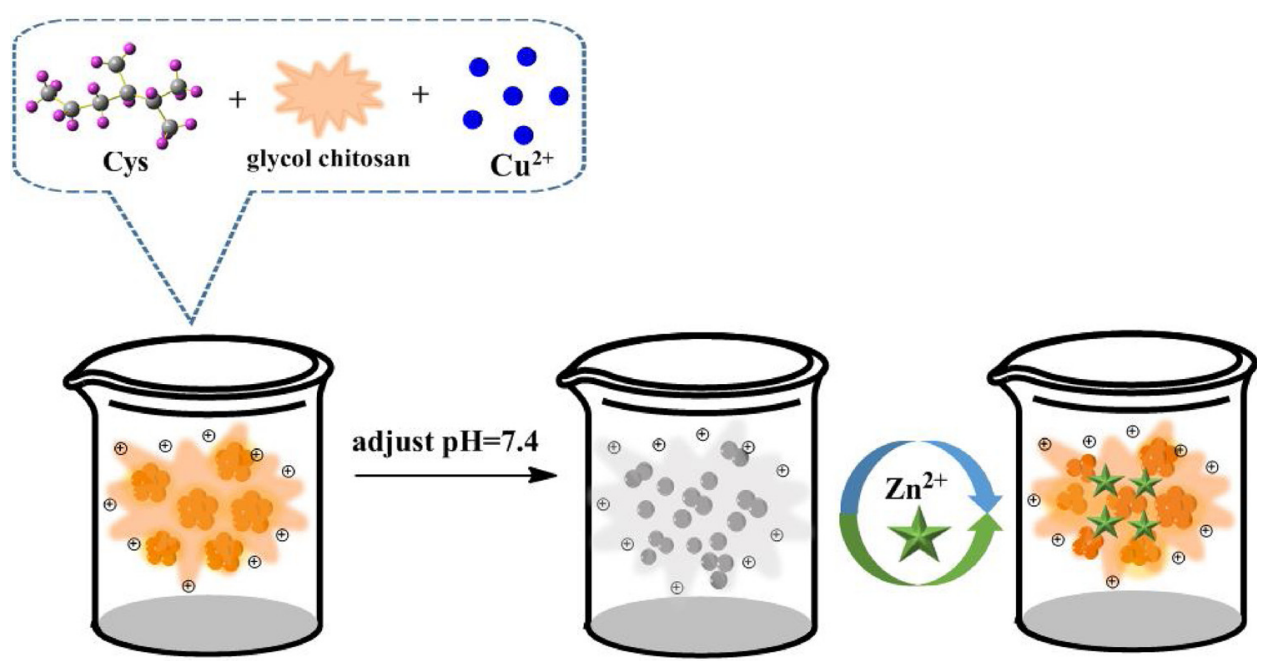

Scheme 1. Schematics showing formation of the CuNCs@GC nanocomposite, and the detection principle of Zn ions.
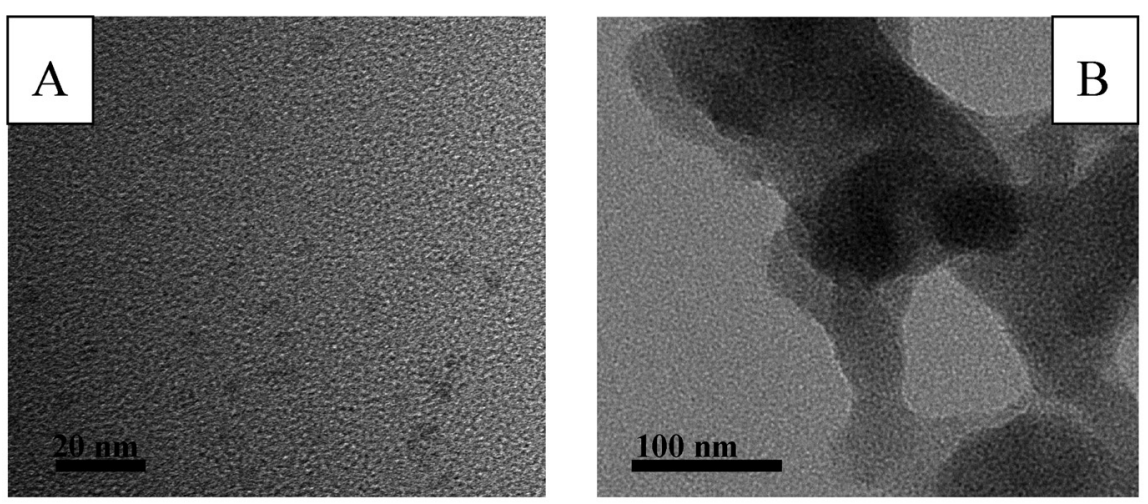

Fig. 1. TEM images of (A) CuNCs and (B) CuNCs@GC (1 mg/mL). (C) The diameter distribution of the CuNCs and CuNCs@GC (1 mg/ mL). (D) Survey XPS spectrum of CuNCs@GC. Inset: XPS spectra of $\mathrm{Cu} 2 \mathrm{p}$ electrons in CuNCs (black line) and CuNCs@GC (red line), the blue dash line represents the binding energy of $\mathrm{Cu}$ (II).(For interpretation of the references to colour in this figure legend, the reader is referred to the web version of this article.)
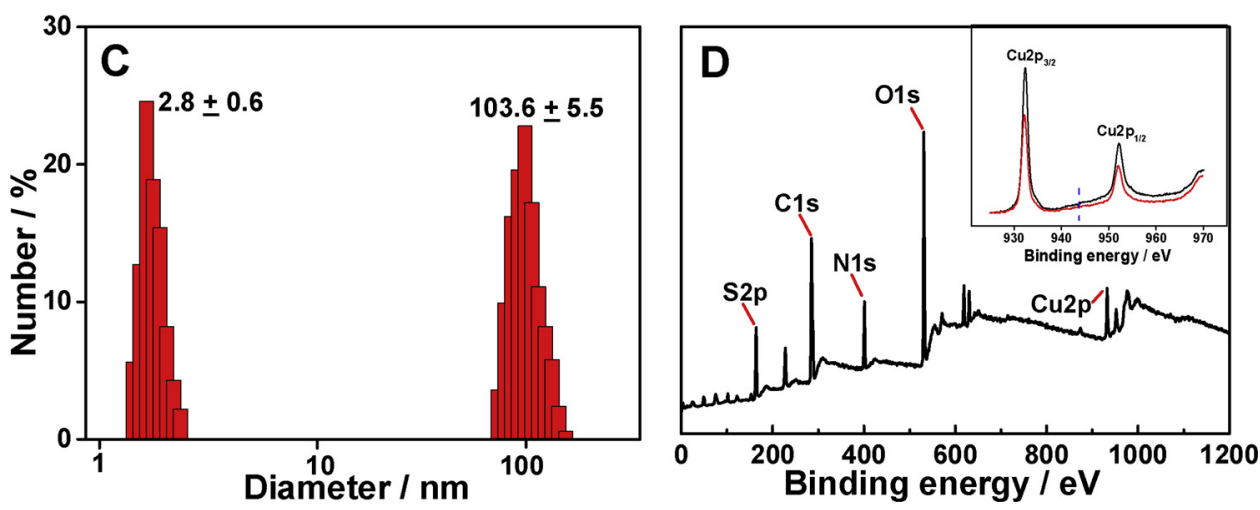

Stokes shift and a reasonably strong PL [14-16]. Compared with those extensively reported precious metal ( $\mathrm{Au}, \mathrm{Ag}$ )-based nanoclusters, $\mathrm{Cu}$ nanoclusters (CuNCs) are cheap to produce from the earth-abundant precursors, low-toxic, and can be easily surface-functionalized by a variety of different ligands, including DNA, amino acids, peptides and thiols [17-21]. Another attractive property of CuNCs is their aggregation induced emission (AIE), the phenomenon which has been first discovered in organic compounds by Tang's group in 2001 and later on reported for metal NCs [22-24]. In particular CuNCs with thiol-containing ligands show pronounced AIE properties, which may provide a powerful tool for analytical chemistry [25-28]. Zhao's group has used glutathione capped CuNCs for detecting $\mathrm{Zn}^{2+}$ and also for imaging $\mathrm{Zn}^{2+}$ in living cells [29]. However, the poor stability of CuNCs has severely limited their applications in vivo and in living cell detection. In addition, the PL of CuNCs may appear as background signals during detection, which result in a poor detection sensitivity.

To tackle these issues, in this work, stable CuNCs were synthesized by impregnating cysteine (Cys) protected CuNCs into the nanogel of glycol chitosan (GC) to form the CuNCs@GC nanocomposite (Scheme 1). Compared with the bare CuNCs, a 3-fold enhancement on the PL intensity was observed for CuNCs@GC, which emitted intense orange light under UV excitation. The stability improvement for CuNCs was attributed to the protective effect of GC against oxidizing species. In addition, the PL intensity of CuNCs@GC could be adjusted by controlling the $\mathrm{pH}$ of the system. A rather weak PL was obtained after adjusting the $\mathrm{pH}$ to 7.4, which could be greatly enhanced after the addition of a trace amount of $\mathrm{Zn}^{2+}$. Based on this concept, a sensitive $\mathrm{Zn}^{2+}$ detection method was proposed, and a linear response between the PL intensity of CuNCs@GC and the concentration of $\mathrm{Zn}^{2+}$, in the range from $1.5 \mu \mathrm{M}$ to $750 \mu \mathrm{M}$, was obtained, with the detection limit being $1.0 \mu \mathrm{M}$. The 

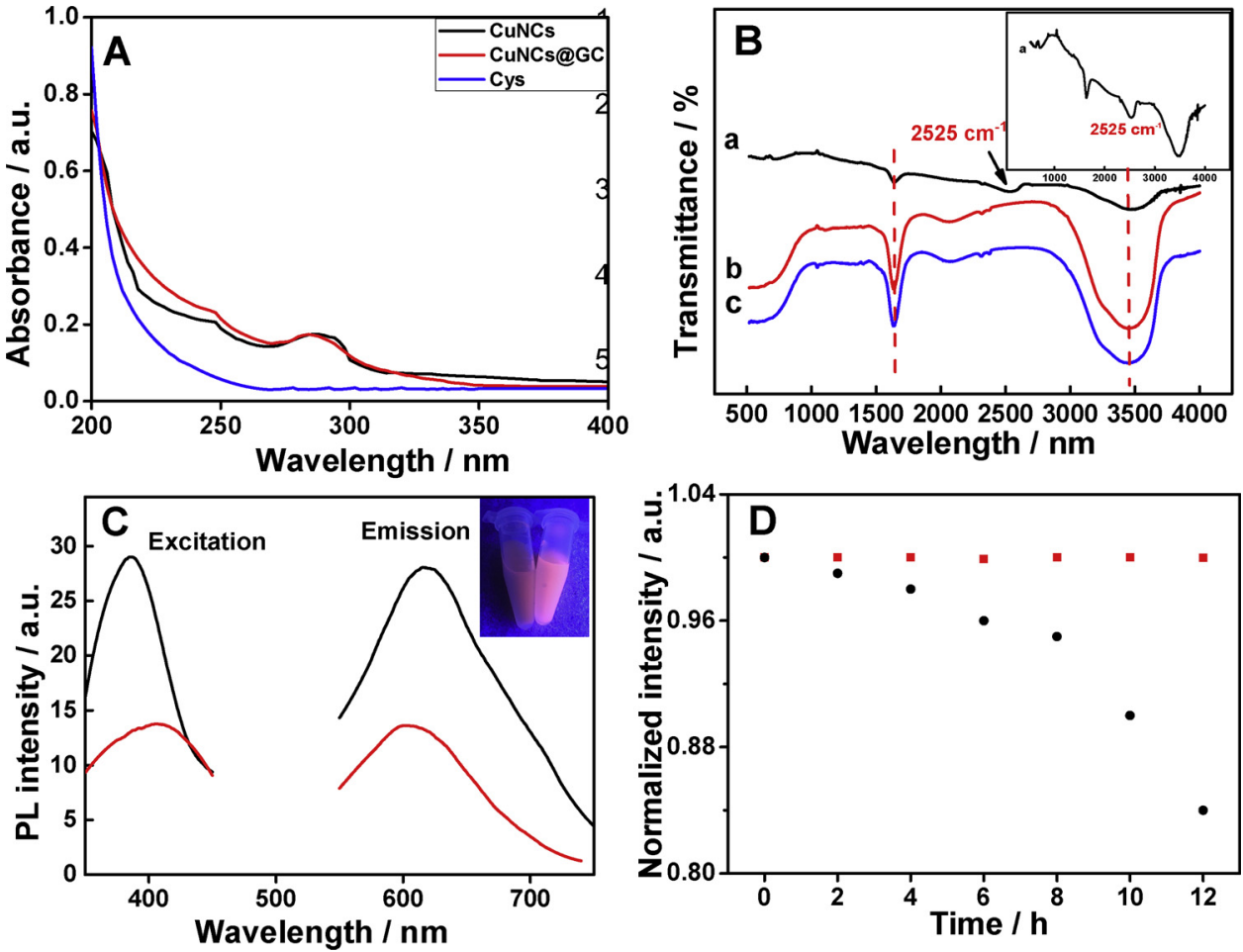

Fig. 2. (A) UV-vis absorption spectra of Cys (blue line), CuNCs (black line) and CuNCs@GC (red line). (B) FT-IR spectra of Cys (a), CuNCs (b) and CuNCs@GC (c). (C) PLE $\left(\lambda_{\mathrm{em}}=605 \mathrm{~nm}\right.$, red line) and PL $\left(\lambda_{\text {ex }}=405 \mathrm{~nm}\right.$, red line) spectra of CuNCs, and PLE $\left(\lambda_{\text {em }}=615 \mathrm{~nm}\right.$, black line $)$ and PL $\left(\lambda_{\text {ex }}=387 \mathrm{~nm}\right.$, black line) spectra of CuNCs@GC. Inset: photographs of CuNCs (left) and CuNCs@GC (right) aqueous solutions under $365 \mathrm{~nm}$ UV irradiation. (D) Change of the PL intensity of CuNCs (black dots) and CuNCs@GC (red dots) with pH = 5 kept under daylight for $12 \mathrm{~h}$. (For interpretation of the references to colour in this figure legend, the reader is referred to the web version of this article.) proposed method was further adapted to detect $\mathrm{Zn}^{2+}$ in living cells, and benefited from high stability, low toxicity and high biocompatibility of CuNCs@GC.

\section{Experimental section}

\subsection{Reagents}

Cys, L-glutathione (GSH), dopamine (DA), uric acid (UA), L-ascorbic acid (AA), chitosan, and GC were purchased from Sigma-Aldrich. Glucose monohydrate, lactate, and $\mathrm{CuSO}_{4} \cdot 5 \mathrm{H}_{2} \mathrm{O}, \mathrm{NaOH}$ were obtained from Sinopharm Chemical Reagent Co., Ltd (Shanghai, China). Amino acids (glycine, alanine, phenylalanine, valine, histidine, etc.) were obtained from Aladdin Chemistry Co., Ltd (Shanghai, China).

\subsection{Instruments}

Transmission electron microscopy (TEM) images were obtained on a JEM-2010 TEM (200 kV). X-ray photoelectron spectroscopy (XPS) data were collected on a Thermo ESCALAB 250XI (USA). UV-vis absorption spectra were recorded on a $2450 \mathrm{UV}$-vis spectrometer (Shimadzu, Japan). Fourier transform infrared (FT-IR) spectra were recorded on a Nicolet 5700 FT-IR spectrometer (USA). A fluorescence spectrophotometer (Cary Eclipse, Varian) was used to collect PL spectra. Dynamic light scattering (DLS) and Zeta potential analysis were carried out on a Zetasizer Nano-ZS90 (Malvern). The humidified incubator (Thermo Scientific 3111) was used for culturing cells.

\subsection{Synthesis of CuNCs}

CuNCs were synthesized by dissolving $0.0605 \mathrm{~g}$ Cys into $5.0 \mathrm{~mL}$ of $\mathrm{CuSO}_{4}$ aqueous solution $(20 \mathrm{mM})$ with ultrasonic treatment, the color of the solution gradually changed from brown to light yellow in the process. The mixed solution was stirring gently for $10 \mathrm{~min}$, and then formed the suspension with an orange emission at room temperature. The obtained suspension was washed with ultrapure water and centrifuged three times at $6000 \mathrm{rpm}$ for $5 \mathrm{~min}$, and the final precipitate was redissolved into $5 \mathrm{~mL}$ ultrapure water and then kept at $4{ }^{\circ} \mathrm{C}$ for further use.

\subsection{Fabrication of CuNCs@GC}

High concentrated GC solution was prepared by dissolving $50 \mathrm{mg}$ GC into $10 \mathrm{~mL}$ of water under water-bath heating for $15 \mathrm{~min}$, and then diluted to different concentration with ultrapure water after cooling to room temperature. Next, $0.1210 \mathrm{~g}$ Cys was added into $2.5 \mathrm{~mL}$ of different concentration of GC solution under ultrasonic treatment, followed by mixing $2.5 \mathrm{~mL}$ of $\mathrm{CuSO}_{4}$ aqueous solution $(40 \mathrm{mM})$. The mixture was kept for 30 min under gentle stirring, and developed an orange-red emission under irradiation of UV light. In the same way, the obtained mixture was washed with ultrapure water and centrifuged three times at $6000 \mathrm{rpm}$ for $5 \mathrm{~min}$, and the final precipitate was redissolved into $5 \mathrm{~mL}$ ultrapure water and then kept at $4{ }^{\circ} \mathrm{C}$ for further use.

\subsection{Procedure for in vitro $\mathrm{Zn}^{2+}$ detection}

The pH of CuNCs and CuNCs@GC was adjusted to 7.4 using $\mathrm{NaOH}$ solution $(1 \mathrm{M})$, respectively. Then, $200 \mu \mathrm{L}$ of $\mathrm{Zn}^{2+}$ solution with different concentrations was added into $400 \mu \mathrm{L}$ of CuNCs and CuNCs@GC aqueous solution, respectively. The mixed solutions were diluted to $1 \mathrm{~mL}$ with ultrapure water, before PL measurements.

\subsection{Cell toxicity assay}

MTT (3-(4,5dimethylthiazolyl)-2,5dimethylthiazol-2-yl)-2,5diphenyltetrazolium bromide) assay was used to assess the cytotoxicity of CuNCs@GC. A549 cells were seeded into a 96-well cell culture plate at a final density of 8000 cells/well. Different volumes of as-prepared CuNCs@GC solution $(1,2.5,5,7.5,10,15 \mu \mathrm{L})$ were added to the wells. The cells were then incubated for $24 \mathrm{~h}$ at $37^{\circ} \mathrm{C}$ under $5 \% \mathrm{CO}_{2}$. Subsequently, MTT was added to each well (final concentration of $5 \mathrm{mg} \mathrm{mL}^{-1}$ ), and the plate was incubated for $4 \mathrm{~h}$ at $37^{\circ} \mathrm{C}$ under $5 \%$ $\mathrm{CO}_{2}$. Then, formazan crystals were dissolved in $150 \mu \mathrm{L}$ of DMSO. The amount of MTT formazan was quantified by measuring the absorbance 

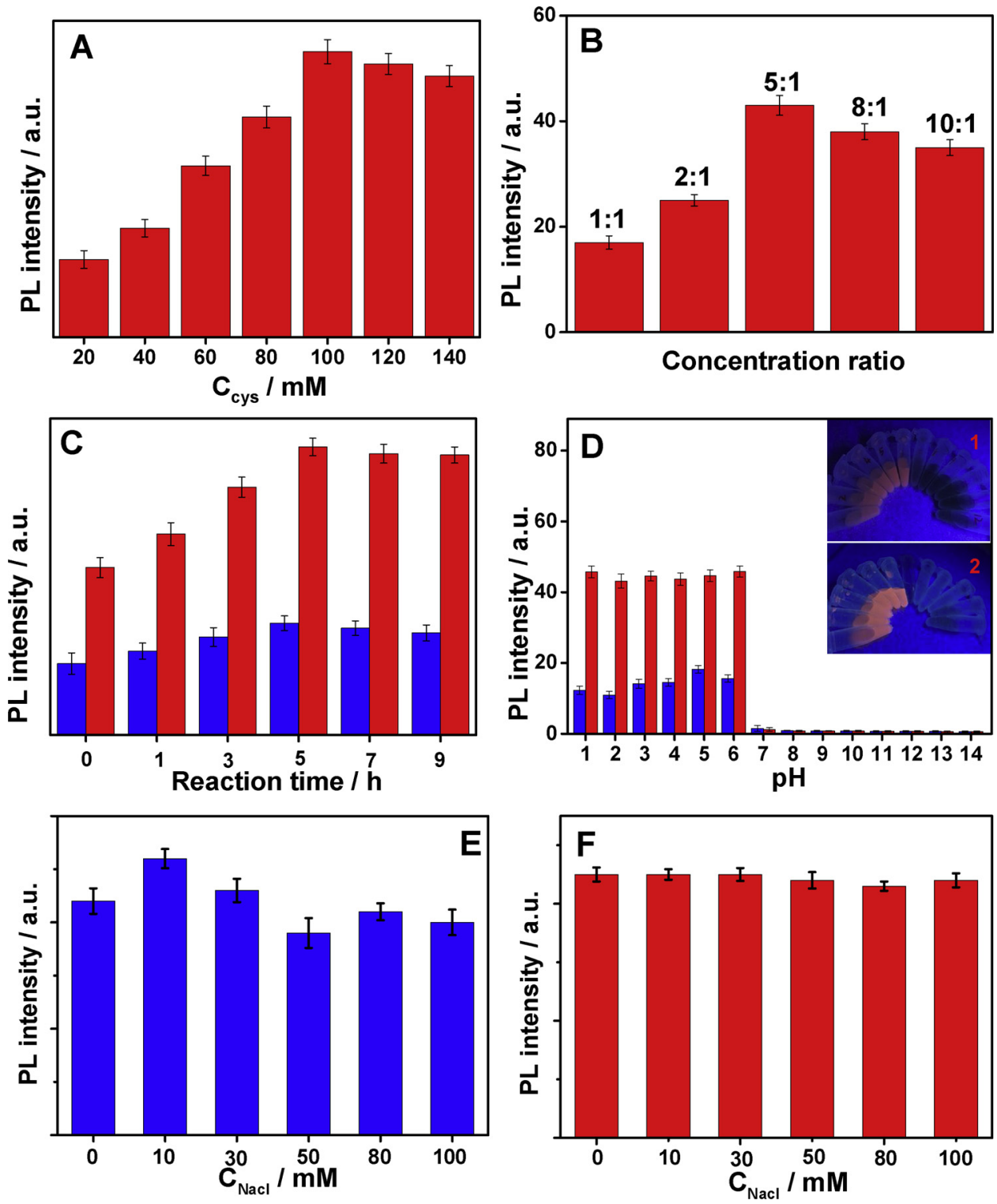

Fig. 3. (A) PL intensity of CuNCs@GC $(\mathrm{pH}=2)$ as a function of the concentration of Cys. (B) PL intensity of CuNCs@GC in dependence of the concentration ratio of $\mathrm{GC}$ and $\mathrm{Cu}^{2+}$. (C) PL intensity of CuNCs (blue line) and CuNCs@GC (red line) as a function of the reaction time. (D) PL intensity of CuNCs (blue line) and CuNCs@GC (red line) as a function of the $\mathrm{pH}$ value. Inset: photographs of CuNCs (1) and CuNCs@GC (2) at different pH values (changing from acidic to basic values from left to right) under $365 \mathrm{~nm} \mathrm{UV}$ irradiation. The stability of the CuNCs (E) and CuNCs@GC (F) in the presence of various concentrations of $\mathrm{NaCl}$ solution. (For interpretation of the references to colour in this figure legend, the reader is referred to the web version of this article.) at $570 \mathrm{~nm}$ using a microplate reader (Tecan, Austria).

\subsection{Living cell imaging}

A549 cells were cultured in a medium with $10 \%$ fetal bovine serum (FBS) and $1 \%$ penicillin/streptomycin $\left(100\right.$ units $\left.\mathrm{mL}^{-1}\right)$ at $37^{\circ} \mathrm{C}$ in a humidified incubator with $5 \% \mathrm{CO}_{2}$ for $24 \mathrm{~h}$. After that, the cells were washed twice with freshly prepared phosphate buffer solution (PBS, $10 \mathrm{mM}, \mathrm{pH}=7.4$ ). The cells were then incubated at $37^{\circ} \mathrm{C}$ for $2 \mathrm{~h}$ in $1 \mathrm{~mL}$ of the cell medium (containing $10 \mu \mathrm{L}$ of CuNCs@GC $\left(5 \mathrm{mg} \mathrm{mL}^{-1}\right)$ solution) mentioned above. For $\mathrm{Zn}^{2+}$ detection, CuNCs@GC solution (containing $500 \mu \mathrm{M}$ of $\mathrm{Zn}^{2+}$ ) was used instead of pure CuNCs@GC solution. After rinsing five times by PBS, cell imaging was carried out with an excitation at $405 \mathrm{~nm}$ under a fluorescence microscope system.

\section{Results and discussion}

\subsection{Morphology characterization of CuNCs and CuNCs@GC}

The TEM image in Fig. 1A shows that CuNCs were dispersed in aqueous solution with an uneven and loose state of aggregation. After impregnated them into $\mathrm{GC}$, which is a biocompatible derivative of chitosan that is soluble at neutral pH, to form CuNCs@GC via the electrostatic interaction, several morphological changes were observed (Fig. S1). Because the isoelectric point of Cys is 5.05, during nanocomposite fabrication, GC first interacted with the negatively charged Cys resulting in an electrostatic binding. Subsequent addition of aqueous $\mathrm{CuSO}_{4}$ solution led to $\mathrm{Cu}-\mathrm{S}$ interactions between copper ions and the pre-formed ionic nanogel for nanocomposite generation. The amount of GC influenced the PL intensity of nanocomposites, and the maximum was observed at the concentration of $1 \mathrm{mg} \mathrm{mL}^{-1}$ (Fig. S2). CuNCs could be coated incompletely with an insufficient amount of GC (Fig. S1A), while an excessive amount of GC would cause severely aggregation of polymer itself (Fig. S1C, D) [17]. Therefore, a proper amount of GC could change the loose aggregation state of CuNCs into the dense aggregation state, which not only promoted the system stability but also enhanced the PL intensity, forming a self-assembled structure (Figs. 1B, S1C).

At the same time, the data of dynamic light scattering (DLS) was recorded to characterize other properties of CuNCs and CuNCs@GC. Fig. 1C displayed that the average size of CuNCs was around $2.8 \pm 0.6 \mathrm{~nm}$, while the average size of CuNCs@GC was increased to around $102.3 \pm 7.9 \mathrm{~nm}$ after CuNCs were impregnated into the GC matrix. The XPS spectrum was used to confirm the composition and 

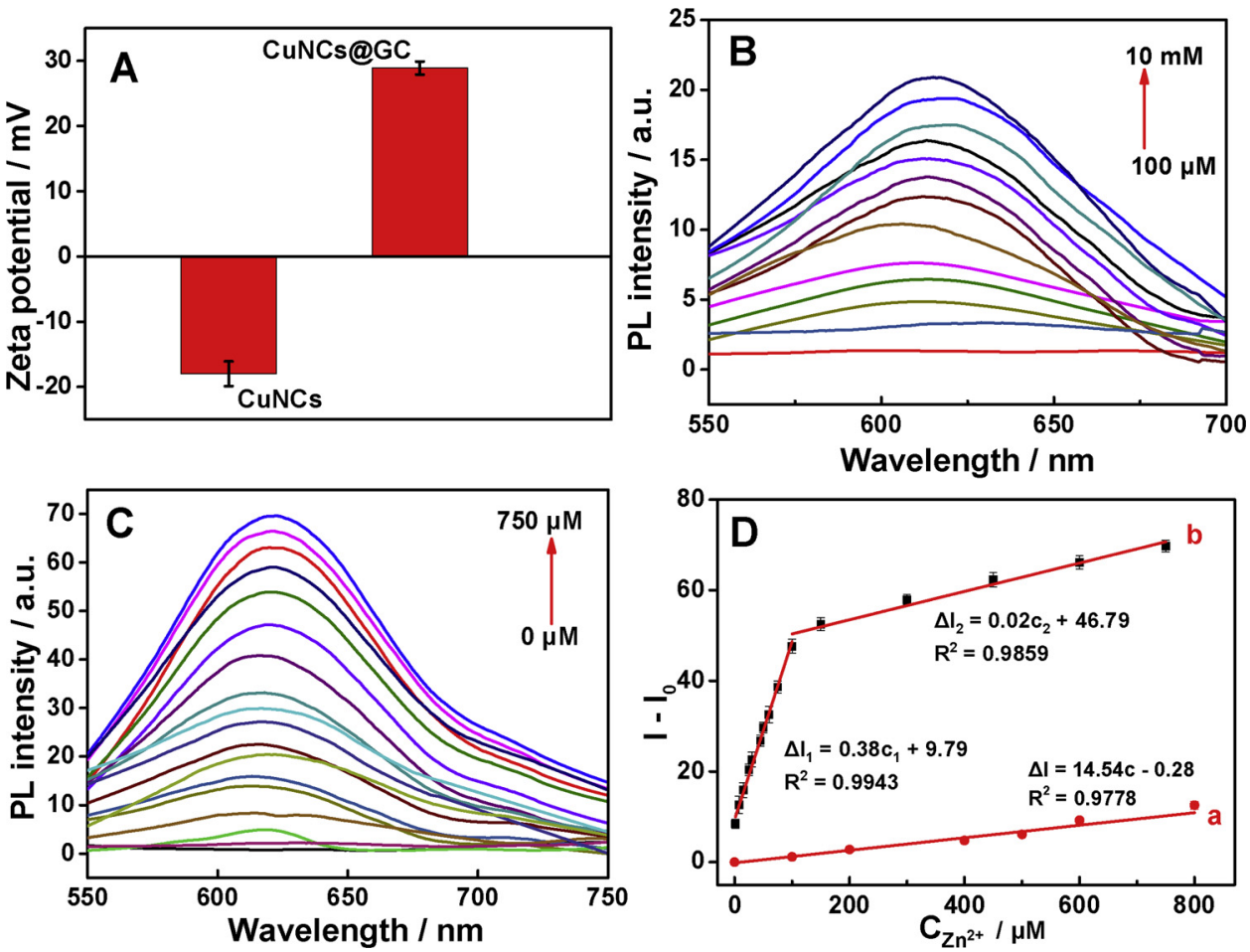

Fig. 4. (A) Zeta potential of CuNCs and CuNCs@GC at $\mathrm{pH}=7.4$. PL spectra of CuNCs (B) and CuNCs@GC (C) after adding $\mathrm{Zn}^{2+}$ in the concentration range of $100 \mu \mathrm{M}-10 \mathrm{mM}$ and $0.1 \mu \mathrm{M}-750 \mu \mathrm{M}$, respectively. (D) Linear relationships between the PL intensity difference and various concentrations of $\mathrm{Zn}^{2+}$ for CuNCs (a) and CuNCs@GC (b). valence state of elements in CuNCs and CuNCs@GC. C, O, N, S, and Cu were observed in CuNCs@GC (Fig. 1D), as well as in CuNCs (Fig. S3A), indicating that the addition of the GC nanogel did not induce change of elements in the CuNCs@GC system. Inset of Fig. 1D showed that the peaks at 932.4 and $952.5 \mathrm{eV}$ were attributed to $\mathrm{Cu} 2 \mathrm{p}_{3 / 2}$ and $\mathrm{Cu} 2 \mathrm{p}_{1 / 2}$ of $\mathrm{Cu}(0)$, respectively, and there was no satellite peak at $943.7 \mathrm{eV}$, indicating that $\mathrm{Cu}(\mathrm{II})$ was almost absent in the system [30]. It should be noted that counts of Cu2p had a difference of $1 \mathrm{mg} \mathrm{mL}^{-1}$ between CuNCs (black line) and CuNCs@GC (red line). This may be related to the electrostatic interaction of CuNCs with GC [22]. As shown in Fig. S3B, with an increasing concentration of GC, the counts of Cu2p were decreased. It was reported that binding energies of $\mathrm{Cu}(0)$ and $\mathrm{Cu}(\mathrm{I})$ differ by $0.1 \mathrm{eV}$ approximately, therefore, the valence of $\mathrm{Cu}$ in the product was most likely between 0 and +1 [23].

Fig. 2A displays the UV-vis absorption spectra of CuNCs (black line) and CuNCs@GC (red line). Both of them showed a wide absorption band at $284 \mathrm{~nm}$. However, pure Cys (blue line) had no characteristic absorption band in $200-300 \mathrm{~nm}$, indicating that $\mathrm{Cu}^{2+}$ could catalyze autoxidation of Cys to form the $\mathrm{Cu}(\mathrm{I})$-Cys complex [31]. The FT-IR spectra were used for molecular structure analysis and identification. As shown in Fig. 2B, the characteristic peak at $2525 \mathrm{~cm}^{-1}$, which was from the $\mathrm{S}-\mathrm{H}$ stretching vibration mode of free Cys, disappeared in the CuNCs (b) and CuNCs@GC (c), suggesting that the Cys as stabilizing ligands was modified on the CuNCs surfaces through $\mathrm{Cu}-\mathrm{S}$ bonding [32].

As shown in Fig. 2C, the excitation and emission of CuNCs (red line) were peaked at $405 \mathrm{~nm}$ and $605 \mathrm{~nm}$ respectively, while CuNCs@GC (black line) showed a strong emission peak at $615 \mathrm{~nm}$ under excitation at $387 \mathrm{~nm}$, indicating that CuNCs embedded in GC not only had a large Stokes shift but were also easier to be excited. Moreover, wrapped GC could protect CuNCs from unwanted relaxation, so that CuNCs@GC emitted saffron yellow light rather than yellow PL under UV irradiation at $365 \mathrm{~nm}$, as shown in the inset of Fig. 2C. PL spectra of CuNCs@GC exhibited different intensities with no significant peak shift under the excitation wavelength of $310-410 \mathrm{~nm}$ in Fig. S4. This indicated that CuNCs@GC obeyed the Kasha-Vavilov rule of excitation independent emission [33]. In order to further clarify the superiority of CuNCs after the incorporation of the GC nanogel, CuNCs and CuNCs@GC were consecutively exposed to UV irradiation and daylight for different times. Fig. 2D shows that the photostability of CuNCs@GC was indeed superior to pure CuNCs after exposure to sunlight for $12 \mathrm{~h}$, as well as to $\mathrm{UV}$ irradiation for $2 \mathrm{~h}$ (Fig. S5). Considering that the $\mathrm{O}_{2}$ might affect the stability of CuNCs and CuNCs@GC, the prepared CuNCs@GC and CuNCs solution were respectively exposed to $\mathrm{N}_{2}$ for $5 \mathrm{~min}$ to remove $\mathrm{O}_{2}$, and then the changes of fluorescence were recorded every two hours. Fig. S6 showed that the stability of the CuNCs was better in the absence or the low level of $\mathrm{O}_{2}$, however, this had little effect on the CuNCs@GC, which was attributed to the protective effect of GC against oxidizing species.

\subsection{Optimization of experimental conditions of the formation of CuNCs@GC composite}

The concentration of Cys, the molar ratio of GC to $\mathrm{Cu}^{2+}$, the type of nanogels, the reaction time, and the $\mathrm{pH}$ of the system were considered and controlled in this work. As shown in Fig. 3, the optimal synthetic conditions for the formation of CuNCs@GC were achieved by reacting $100 \mathrm{mM} \mathrm{Cys} 1 \mathrm{mg} \mathrm{mL}^{-1} \mathrm{GC}$ and $20 \mathrm{mM} \mathrm{Cu}^{2+}$ (molar ratio of GC to $\mathrm{Cu}^{2+}$ of 5:1) for $5 \mathrm{~h}$. Interestingly, the fluorescence intensity of CuNCs and CuNCs@GC was different under different pH value, Fig. 3D displayed that there was no fluorescence signal was generated from the system when the pH value of the CuNCs and CuNCs@GC solution kept in the range 7.0-14.0, while they exhibited a strong fluorescence when the $\mathrm{pH}$ was in the acidic range. We firstly investigated the stability of $\mathrm{GC}$ and chitosan under full range of $\mathrm{pH}$ values (Fig. S7). Compared with GC solution, the chitosan solution turned into a white flocculent suspension under alkaline $\mathrm{pH}$, because chitosan is insoluble in water under alkaline conditions [14]. It is assumed that the main force for the formation of the CuNCs@GC nanocomposite under acidic conditions was came from the strong electrostatic interaction between GC polymer and ligands of $\mathrm{Cu}-\mathrm{S}$ complexes, and the reason for the strong electrostatic interaction is that most of the amine groups of GC are protonated under this conditions. When the $\mathrm{pH}$ increased to alkaline conditions, it has been reported that the amine groups of GC are fully deprotonated, and the CuNCs was existed in CuNCs@GC solution with the dispersed state, resulting in the photoluminescence was disappeared. Therefore, the 

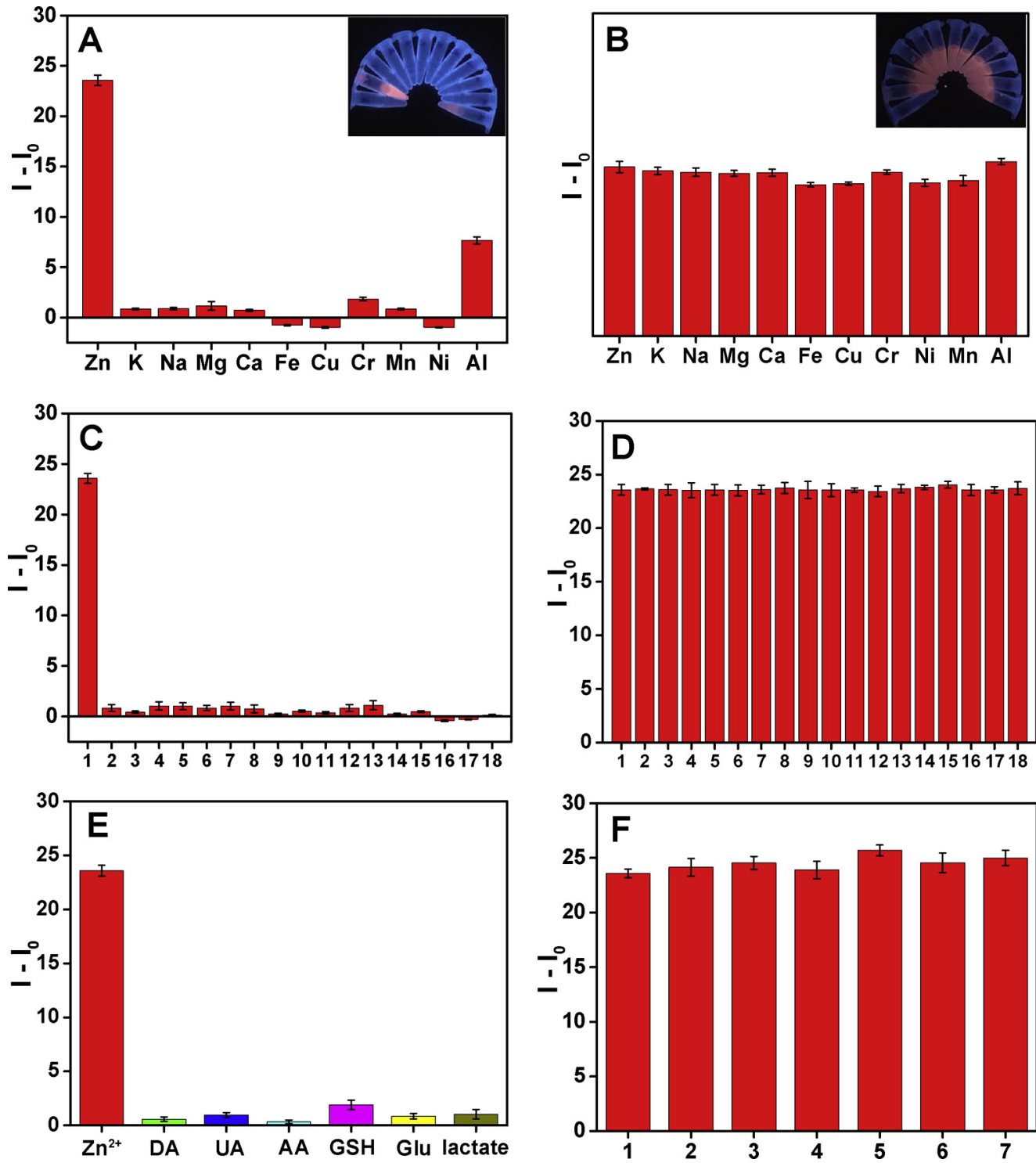

Fig. 5. (A, C, E) Selectivity and (B, D, E) interference of CuNCs@GC toward different physiological substances. (A) Selectivity of metal ions against $\mathrm{Zn}^{2+}$ $(30 \mu \mathrm{M})$, and (B) competition experiments in the presence of $\mathrm{Zn}^{2+}(30 \mu \mathrm{M})$ with the coexistence of interfering ions: $\mathrm{Zn}^{2+}, \mathrm{K}^{+}, \mathrm{Na}^{+}, \mathrm{Mg}^{2+}, \mathrm{Ca}^{2+}, \mathrm{Fe}^{3+}$, $\mathrm{Cu}^{2+}, \mathrm{Cr}^{3+}, \mathrm{Mn}^{2+}, \mathrm{Ni}^{2+}, \mathrm{Al}^{3+}$. The concentrations of ions are $300 \mu \mathrm{M}$, except for $\mathrm{Ar}^{3+}$ (which is $30 \mu \mathrm{M}$ ) and $\mathrm{K}^{+}$, $\mathrm{Na}^{+}, \mathrm{Mg}^{2+}, \mathrm{Ca}^{2+}$ (which are $1.5 \mathrm{mM}$ ). (C) Selectivity of amino acids against $\mathrm{Zn}^{2+}(30 \mu \mathrm{M})$, and (D) competition experiments in the presence of $\mathrm{Zn}^{2+}$ $(30 \mu \mathrm{M})$ with the coexistence of interferences: $1 \mathrm{Zn}^{2+}, 2$ glycine, 3 alanine, 4 phenylalanine, 5 valine, 6 histidine, 7 isoleucine, 8 arginine, 9 aspartic acid, 10 proline, 11 serine, 12 leucine, 13 tyrosinase, 14 tryptophan, 15 threonine, 16 asparagine, 17 glutamic and 18 methionine. The concentrations of all amino acids are $300 \mu \mathrm{M}$. (E) Selectivity of biological species against $\mathrm{Zn}^{2+}$ and (F) competition experiments in the presence of $\mathrm{Zn}^{2+}$ with the coexistence of interfering substances: $\mathrm{Zn}^{2+}(30 \mu \mathrm{M}), \mathrm{DA}, \mathrm{UA}$ and AA (1.5 mM), GSH $(600 \mu \mathrm{M})$, glucose (3 $\mathrm{mM})$, lactate $(3 \mathrm{mM})$.
CuNCs@GC nanocomposite had strong PL intensity under acidic conditions $[17,34,35]$. Considering that $\mathrm{pH} 7.4$ is the optimal $\mathrm{pH}$ for biological systems, we selected pH 7.0 as preferential experimental condition. The CuNCs@GC were subjected to pH cycling between pH 5.0 and 9.0 to evaluate the reversible response to the change of $\mathrm{pH}$ value. As shown in Fig. S8, the fluorescence intensity of CuNCs@GC was relatively stable after five consecutive repeated cycles, indicating that the CuNCs@GC displayed well reversible response toward pH value. In addition, the stability of CuNCs and CuNCs@GC under salinity conditions was investigated with different concentrations of $\mathrm{NaCl}$ in this work. As shown in Fig. 3E and F, the fluorescence intensity of CuNCs@GC remained almost unchangeable even the salt-containing solutions was up to 100 mM. However, compared with the CuNCs@GC, the PL intensity of CuNCs without GC showed an obvious fluctuation trend to some extent, indicating that the CuNCs@GC possessed excellent stability in a high ionic strength medium.

\subsection{In vitro detection of $\mathrm{Zn}^{2+}$}

Considering GC holds a large amount of amine groups, which may cause the charge on the surface of CuNCs@GC to be different from CuNCs, it is expected to be able to further integrate it with $\mathrm{Zn}^{2+}$. As shown in Fig. 4A, the zeta potential of CuNCs was $-17.8 \mathrm{mV}$ at
$\mathrm{pH}=7.4$, which revealed that CuNCs were negatively charged and the electrostatic repulsion led by the carboxylate ions $\left(\mathrm{COO}^{-}\right)$of Cys protected them from aggregation. The zeta potential of CuNCs@GC, on the other hand, was positive, namely $28.9 \mathrm{mV}$, suggesting that the inversion of overall charge through electrostatic interactions [17]. The zeta potential of CuNCs@GC in Fig. S9 experienced an obvious change with the increasing concentration of $\mathrm{Zn}^{2+}$ increasing from $28.9 \mathrm{mV}$ to $35.1 \mathrm{mV}$, which suggests that $\mathrm{Zn}^{2+}$ could combine with $\mathrm{COO}^{-}$on the surface of CuNCs@GC. Importantly, as a cross-linker, Zn ${ }^{2+}$ changed CuNCs@GC from the dispersed state to the aggregated state, so that its PL intensity became enhanced (Fig. S10A). TEM image illustrated an aggregation of CuNCs@GC in the presence of $\mathrm{Zn}^{2+}$ (Fig. S10B), and the emission intensity greatly enhanced after the addition of $\mathrm{Zn}^{2+}$ in the comparison of CuNCs@GC solution, which might be related to that the addition of $\mathrm{Zn}^{2+}$ could lead to a density change of agglomerates, resulting in a different degree of scattering after being excited. In addition, upon addition of $\mathrm{Zn}^{2+}$, there was no obvious change in the UV-vis absorption and FT-IR spectra of CuNCs@GC as compared with pure CuNCs@GC (Fig. S10C, D). This suggested that $\mathrm{Zn}^{2+}$ ions were not involved in any chemical reaction with CuNCs@GC, but rather re-aggregated dispersed nanoclusters in this composite, as a cross-linker. The above observations indicated that $\mathrm{Zn}^{2+}$ are able to cause the AIE effect of CuNCs@GC, similar to the findings in previous reports $[29,36]$. 

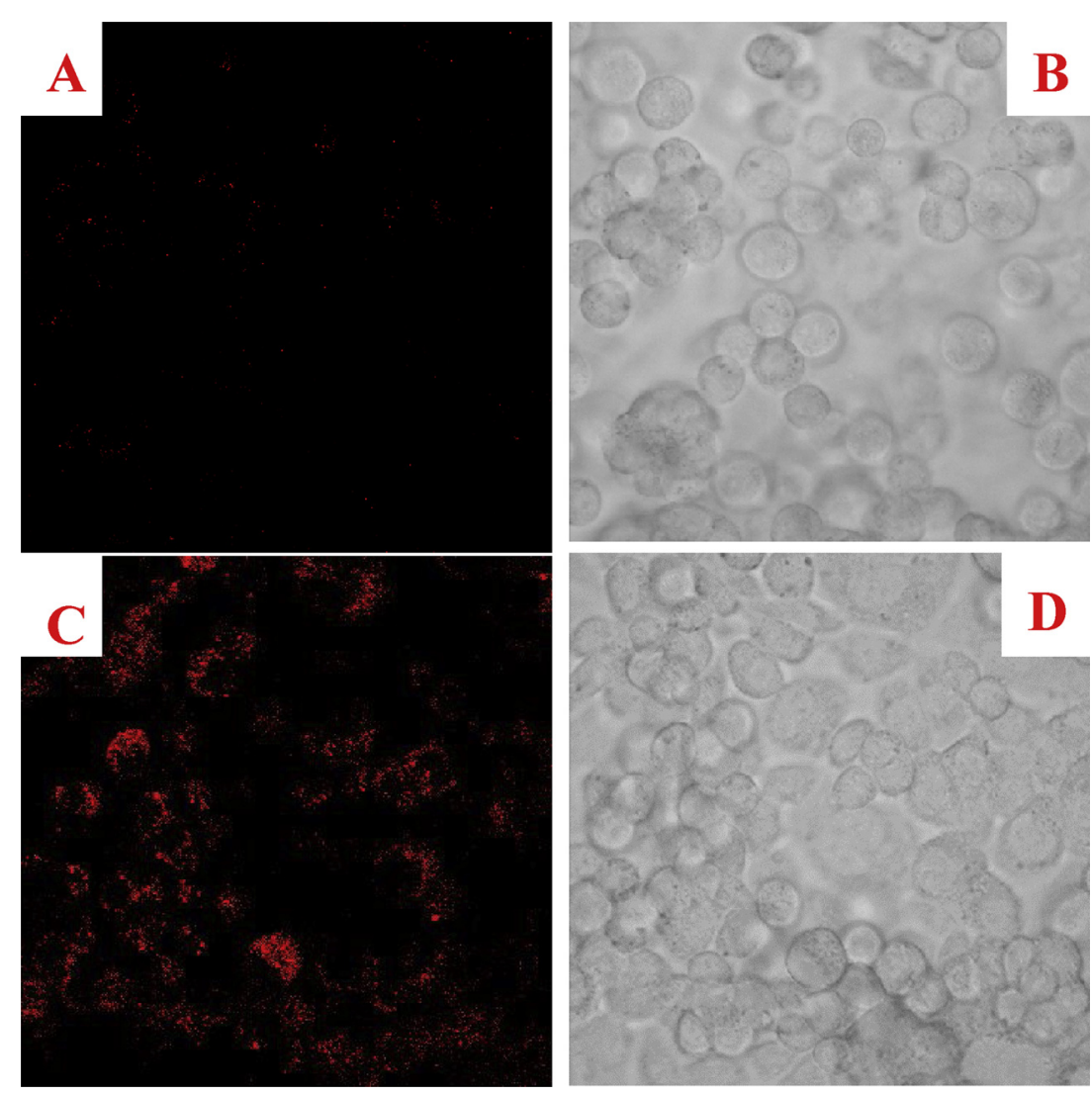

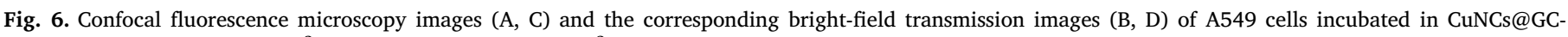
containing medium without $\mathrm{Zn}^{2+}(\mathrm{A}, \mathrm{B})$ and with $500 \mu \mathrm{M} \mathrm{Zn}{ }^{2+}(\mathrm{C}, \mathrm{D})$.

Under establishing optimal formation conditions, changes of $\mathrm{PL}$ intensity of CuNCs@GC were studied with the addition of different concentrations of $\mathrm{Zn}^{2+}$. The evolution of PL spectra of CuNCs was also tested for comparison. As shown in Fig. 4B and C, the PL intensities of CuNCs and CuNCs@GC were enhanced when $\mathrm{Zn}^{2+}$ was added at a concentration of $100 \mu \mathrm{M}-10 \mathrm{mM}$, and $0.1 \mu \mathrm{M}-750 \mu \mathrm{M}$, respectively. Fig. 4D shows linear relationships with the concentration of $\mathrm{Zn}^{2+}$ for both CuNCs (a) and CuNCs@GC (b).CuNCs@GC was found to be more sensitive than CuNCs for the detection of $\mathrm{Zn}^{2+}$ : The detection limit of CuNCs was about $75 \mu \mathrm{M}(\mathrm{S} / \mathrm{N}=3)$ for $\mathrm{Zn}^{2+}$ in the range from $100 \mu \mathrm{M}$ to $10 \mathrm{mM}$, while the detection limit for CuNCs@GC was $1.0 \mu \mathrm{M}$ in the linear concentration from $1.5 \mu \mathrm{M}$ to $750 \mu \mathrm{M}$, which is far lower than $76 \mu \mathrm{M}$, the permitted concentration of $\mathrm{Zn}^{2+}$ as set by World Health Organization, in drinking water [37]. The linear regression equation for CuNCs was $\Delta I=14.54 c-0.28\left(\Delta I=I-I_{O}\right.$, where $I$ represents the PL intensity in the presence of $\mathrm{Zn}^{2+}, I_{O}$ represents the PL intensity of CuNCs at $\mathrm{pH}=7.4$, and $c$ represents the concentration of $\mathrm{Zn}^{2+}$ ) in the concentration range from 0 to $800 \mu \mathrm{M}$, and for CuNCs@GC, the linear regression equations were $\Delta I_{1}=0.38 c_{1}-9.97(1.5-100 \mu \mathrm{M})$ and $\Delta I_{2}=0.02 c_{2}+46.79(100-750 \mu \mathrm{M})$. We also examined the change of

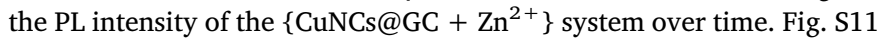

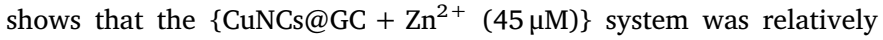
stable within three $\mathrm{h}$, while the $\left\{\mathrm{CuNCs}+\mathrm{Zn}^{2+}(1 \mathrm{mM})\right\}$ system was found unstable for that period of time. The evolution of the PL spectra of the three different systems with $\mathrm{Zn}^{2+}$ in the concentration range from 0 to $300 \mu \mathrm{M}$ is shown in Fig. S12, from which the superiority of CuNCs@GC was evident compared to both CuNCs and chitosan-coated CuNCs. This means that GC-capped CuNCs are well protected from structural looseness and relaxation, thus effectively increasing the contact area with $\mathrm{Zn}^{2+}$ and remaining stable [38,39]. The AIE effect of CuNCs@GC was then able to be triggered by $\mathrm{Zn}^{2+}$ as a cross-linker, resulting in the strong and stable PL intensity.
The selectivity of the CuNCs@GC sensing system to $\mathrm{Zn}^{2+}$ was also tested for various species that may interfere with the detection of $\mathrm{Zn}^{2+}$, including metal ions, amino acids, and several other biological substances. For metal ions, those commonly found in living organism $\left(\mathrm{K}^{+}\right.$, $\mathrm{Na}^{+}, \mathrm{Ca}^{2+}, \mathrm{Mg}^{2+}$ ) that are 50 times higher in concentration than that of $\mathrm{Zn}^{2+}(30 \mu \mathrm{M})$, and the trace metal ions $\left(\mathrm{Fe}^{3+}, \mathrm{Cu}^{2+}, \mathrm{Cr}^{3+}, \mathrm{Mn}^{2+}\right.$, $\mathrm{Ni}^{2+}, \mathrm{Al}^{3+}$ ) that exist in living cells and are 3-10 times higher in concentration than that of $\mathrm{Zn}^{2+}(30 \mu \mathrm{M})$ except for $\mathrm{Al}^{3+}$ were separately added into the CuNCs@GC and CuNCs@GC + $\mathrm{Zn}^{2+}$ systems for evaluation. As shown in Fig. 5A, although the same concentration of $\mathrm{Al}^{3+}$ ions could also cause the enhancement of PL intensity, $\mathrm{Zn}^{2+}$ ions could cause significant enhancement of the PL intensity of the CuNCs@GC. Fig. 5B shows that when $\mathrm{Zn}^{2+}$ and other metal ions coexisted in the CuNCs@GC system, those interfering ions had little influence on the efficiency of $\mathrm{Zn}^{2+}$ sensing. Next, amino acids were investigated as interfering substances; Fig. $5 \mathrm{C}$ shows that they did not cause enhancement of the PL intensity of the CuNCs@GC system. When $\mathrm{Zn}^{2+}$ coexisted with amino acids (Fig. 5D), the PL intensity of the CuNCs@GC system was almost the same as the one where only $\mathrm{Zn}^{2+}$ was present. As other biomolecules (such as DA, UA, AA, GSH, glucose and lactate) may interfere with $\mathrm{Zn}^{2+}$ detection, they were tested as well. Fig. 5E and $\mathrm{F}$ shows that the effects of these biological substances on the efficiency of $\mathrm{Zn}^{2+}$ detection were almost negligible.

Table S1 demonstrates that the probe based on CuNCs@GC nanocomposites has a better sensitivity and a wider detection range for $\mathrm{Zn}^{2+}$ detection, compared with other reported probes based on PL materials [40-45]. This makes it a promising sensor for applications in $\mathrm{Zn}^{2+}$ detection in living cells.

\subsection{Application of CuNCs@GC in live cell imaging}

The cytotoxicity of CuNCs@GC was assessed by using the MTT 
method before live cell imaging. More than $90 \%$ of the cells survived after being treated with CuNCs@GC solution (Fig. S13). Fig. 6 displays the microscopic images of the $\mathrm{A} 549$ cells treated with $\mathrm{Zn}^{2+}$. Compared with the images of cells incubated in the absence of $\mathrm{Zn}^{2+}$ (Fig. 6A), the brightness of the cells incubated with $\mathrm{Zn}^{2+}$ was much higher (Fig. $6 \mathrm{C}$ ), which shows that the CuNCs@GC is an excellent candidate for detecting $\mathrm{Zn}^{2+}$ in living cells. Neither observable morphological changes nor signs of cell damage (including cell detachment from the bottom as well as cell debris) were detected for cells after incubation with this nanoprobe, which further confirmed the negligible cytotoxicity of CuNCs@GC during live cell imaging.

\section{Conclusions}

In summary, a PL method for $\mathrm{Zn}^{2+}$ detection was developed, based on the $\mathrm{Zn}^{2+}$-triggered AIE effect of CuNCs@GC, resulting in their transition from the dispersed state to the aggregated state and thus the enhancement of the PL intensity. The large amount of amine groups of GC not only had a significant effect on the structural stability and PL enhancement of CuNCs, but also effectively stabilized the system via crosslinking between $\mathrm{Zn}^{2+}$ and CuNCs. Compared with other reported PL methods, the "turn-on" mode for the $\mathrm{Zn}^{2+}$ detection was demonstrated, with low toxicity, good biocompatibility, good sensitivity, and good selectivity. Moreover, the nanoprobe based on CuNCs@GC was successfully applied to the detection of $\mathrm{Zn}^{2+}$ in live cell imaging. It is expected that this "turn-on" PL nanoprobe for $\mathrm{Zn}^{2+}$ detection could have great application prospects in securing drinking water safety and performing biological analysis.

\section{Declaration of Competing Interest}

There are no conflicts to declare.

\section{Acknowledgements}

This work was financially supported by the National Natural Science Foundation of China (21778047, 21804030), the Hong Kong Scholars Program (XJ2017052), the Science Technology and Innovation Committee of Shenzhen Municipality (JCYJ20170818104224667), and Taishan Scholar Project Special Funding (No. ts20190962).

\section{Appendix A. Supplementary data}

Supplementary material related to this article can be found, in the online version, at doi:https://doi.org/10.1016/j.snb.2019.127626.

\section{References}

[1] A.S. Prasad, Discovery of human zinc deficiency: its impact on human health and disease, Adv. Nutr. 4 (2013) 176-190.

[2] T. Fukada, S. Yamasaki, K. Nishida, M. Murakami, T. Hirano, Zinc homeostasis and signaling in health and diseases, J. Biol. Inorg. Chem. 16 (2011) 1123-1134.

[3] E. Mocchegiani, C. Bertonifreddari, F. Marcellini, M. Malavolta, Brain, aging and neurodegeneration: role of zinc ion availability, Prog. Neurobiol. 75 (2005) 367-390.

[4] B. Szewczyk, M. Kubera, G. Nowak, The role of zinc in neurodegenerative inflammatory pathways in depression, Prog. Neuro-Psychopharmacol. 35 (2011) 693-701.

[5] Q. Li, X. Zhao, Q. Lv, G. Liu, The determination of zinc in water by flame atomic absorption spectrometry after its separation and preconcentration by malachite green loaded microcrystalline triphenylmethane, Sep. Purif. Technol. 55 (2007) $76-81$.

[6] K.S. Rao, T. Balaji, T.P. Rao, Y. Babu, G.R.K. Naidu, Determination of iron, cobalt, nickel, manganese, zinc, copper, cadmium and lead in human hair by inductively coupled plasma-atomic emission spectrometry, Spectrochim. Acta B 57 (2002) $1333-1338$

[7] G. Gumus, H. Filik, B. Demirata, Determination of bismuth and zinc in pharmaceuticals by first derivative UV-visible spectrophotometry, Anal. Chim. Acta 547 (2005) 138-143.

[8] C. Zhang, J. Miura, Y. Nagaosa, Determination of cadmium, zinc, nickel and cobalt in tobacco by reversed-phase high-performance liquid chromatography with 2-(8-
Quinolylazo)-4,5-diphenylimidazole as a chelating reagent, Anal. Sci. 21 (2005) 1105-1110.

[9] D. Nikolelis, G. Raftopoulou, N. Psaroudakis, G.P. Nikoleli, Development of an electrochemical chemosensor for the rapid detection of zinc based on air stable lipid films with incorporated calix4arene phosphoryl receptor, Electroanalysis 20 (2010) 1574-1580.

[10] X. Ji, J. Ren, Determination of copper and zinc in serum by derivative atomic absorption spectrometry using the microsampling technique, Analyst 127 (2002) 416-419.

[11] J.Y. Yoon, S.K. Kim, N.J. Singh, K.S. Kim, Imidazolium receptors for the recognition of anions, Chem. Soc. Rev. 35 (2006) 355-360.

[12] C. Boonmee, V. Promarak, T. Tuntulani, W. Ngeontae, Cysteamine-capped copper nanoclusters as a highly selective turn-on fluorescent assay for the detection of aluminum ions, Talanta 178 (2017) 796-804.

[13] H. Wang, Z. Wang, Y. Xiong, S.V. Kershaw, T. Li, Y. Wang, Y. Zhai, A.L. Rogach, Hydrogen peroxide assisted synthesis of highly luminescent sulfur quantum dots, Angew. Chem. Int. Ed. 58 (2019) 7040-7044.

[14] H. Cao, Z. Chen, H. Zheng, Y. Huang, Copper nanoclusters as a highly sensitive and selective fluorescence sensor for ferric ions in serum and living cells by imaging, Biosens. Bioelectron. 62 (2014) 189-195.

[15] T. Luo, S. Zhang, Y. Wang, M. Wang, M. Liao, X. Kou, Glutathione-stabilized Cu nanocluster-based fluorescent probe for sensitive and selective detection of $\mathrm{Hg}^{2+}$ in water, Luminescence 32 (2017) 1092-1099.

[16] X. Jia, X. Yang, J. Li, D. Li, E. Wang, Stable Cu nanoclusters: from an aggregationinduced emission mechanism to biosensing and catalytic applications, Chem. Commun. 50 (2014) 237-239.

[17] X. Bai, S. Xu, L. Wang, Full-range pH stable Au-clusters in nanogel for confinementenhanced emission and improved sulfide sensing in living cells, Anal. Chem. 90 (2018) 3270-3275.

[18] K. Shanmugaraj, S.A. John, Inner filter effect based selective detection of picric acid in aqueous solution using green luminescent copper nanoclusters, New J. Chem. 42 (2018) 7223-7229.

[19] X. Hu, X. Liu, X. Zhang, H. Chai, Y. Huang, One-pot synthesis of the CuNCs/ZIF-8 nanocomposites for sensitively detecting $\mathrm{H}_{2} \mathrm{O}_{2}$, and screening of oxidase activity, Biosens. Bioelectron. 105 (2018) 65-70.

[20] Z. Wang, R. Chen, Y. Xiong, K. Cepe, J. Schneider, R. Zboril, C.S. Lee, A.L. Rogach, Chemical sensing: incorporating copper nanoclusters into metal-organic frameworks: confinement-assisted emission enhancement and application for trinitrotoluene detection, Part. Part. Syst. Charact. 34 (2017) 1-6.

[21] Z. Wang, Y. Xiong, S.V. Kershaw, B. Chen, X. Yang, N. Goswami, W. Lai, J. Xie, A.L. Rogach, In situ fabrication of flexible, thermally stable, large-area, strongly luminescent copper nanocluster/polymer composite films, Chem. Mater. 29 (2017) 10206-10211.

[22] A. Qin, J.W.Y. Lam, B. Tang, Luminogenic polymers with aggregation-induced emission characteristics, Prog. Polym. Sci. 37 (2012) 182-209.

[23] H. Tong, Y. Hong, Y. Dong, H. Matthias, Z. Li, J.W.Y. Lam, Y. Dong, B. Tang, Protein detection and quantitation by tetraphenylethene-based fluorescent probes with aggregation-induced emission characteristics, J. Phys. Chem. B 111 (2007) 11817-11823.

[24] Z. Zhao, S. Chen, X. Shen, F. Mahtab, Y. Yu, P. Lu, J.W.Y. Lam, H.S. Kwok, B. Tang, Aggregation-induced emission, self-assembly, and electroluminescence of 4,4'-bis (1,2,2-triphenylvinyl)biphenyl, Chem. Commun. 46 (2010) 686-688.

[25] M. Zhao, Z. Qian, M. Zhong, Z. Chen, H. Ao, H. Feng, Fabrication of stable and luminescent copper nanocluster-based AIE particles and their application in $\beta$-galactosidase activity assay, ACS Appl. Mater. Interface 9 (2017) 32887-32895.

[26] Y. Zhong, J. Zhu, Q. Wang, Y. He, Copper nanoclusters coated with bovine serum albumin as a regenerable fluorescent probe for copper(II) ion, Microchim. Acta 182 (2015) 909-915.

[27] X. Liao, R. Li, Z. Li, X. Sun, Z. Wang, J. Liu, Fast synthesis of copper nanoclusters through the use of hydrogen peroxide additive and their application for the fluorescence detection of $\mathrm{Hg}^{2+}$ in water samples, New J. Chem. 39 (2015) 5240-5248.

[28] Z. Wang, Y. Shi, X. Yang, Y. Xiong, Y. Li, B. Chen, W. Lai, A.L. Rogach, Watersoluble biocompatible copolymer hypromellose grafted chitosan able to load exogenous agents and copper nanoclusters with aggregation-induced emission, Adv. Funct. Mater. 28 (2018) 1-7.

[29] L. Lin, Y. Hu, L. Zhang, Y. Huang, S. Zhao, Photoluminescence light-up detection of zinc ion and imaging in living cells based on the aggregation induced emission enhancement of glutathione-capped copper nanoclusters, Biosens. Bioelectron. 94 (2017) 523-529.

[30] N. Goswami, A. Giri, M.S. Bootharaju, P.L. Xavier, T. Pradeep, S.K. Pal, Copper quantum clusters in protein matrix: potential sensor of $\mathrm{Pb}^{2+}$ ion, Anal. Chem. 24 (2011) 9676-9680.

[31] A. Rigo, A. Corazza, M.L.D. Paolo, M. Rossetto, R. Ugolini, M. Scarpa, Interaction of copper with cysteine: stability of cuprous complexes and catalytic role of cupric ions in anaerobic thiol oxidation, J. Inorg. Biochem. 98 (2004) 1495-1501.

[32] T. Zhou, W. Xu, Q. Yao, T. Zhao, X. Chen, Highly fluorescent copper nanoclusters as a probe for the determination of pH, Methods Appl. Fluoresc. 3 (2015) 044002.

[33] A.P. Demchenko, V.I. Tomin, P.T. Chou, Breaking the kasha rule for more efficient photochemistry, Chem. Rev. 21 (2017) 13353-13381.

[34] A.A.P. Mansur, H.S. Mansur, Quantum dot/glycol chitosan fluorescent nanoconjugates, Nanoscale Res. Lett. 10 (2015) 171-179.

[35] C. Wang, C. Wang, L. Xu, H. Cheng, Q. Lin, C. Zhang, Protein-directed synthesis of pH-responsive red fluorescent copper nanoclusters and their applications in cellular imaging and catalysis, Nanoscale 6 (2014) 1775-1781.

[36] D. Li, Z. Chen, Z. Wan, T. Yang, H. Wang, X. Mei, One-pot development of water soluble copper nanoclusters with red emission and aggregation induced 
fluorescence enhancement, RSC Adv. 6 (2016) 34090-34095.

[37] G.J. Park, H. Kim, J.J. Lee, Y.S. Kim, S.Y. Lee, S. Lee, I. Noh, C. Kim, A highly selective turn-on chemosensor capable of monitoring $\mathrm{Zn}^{2+}$ concentrations in living cells and aqueous solution, Sens. Actuators B: Chem. 215 (2015) 568-576.

[38] J. Wang, Y. Kuo, Preparation of fructose-mediated (polyethylene glycol/chitosan) membrane and adsorption of heavy metal ions, J. Appl. Polym. Sci. 105 (2007) 1480-1489.

[39] M.D. Cathell, J.C. Szewczyk, F.A. Bui, C.A. Weber, J.D. Wolever, J. Kang, C.L. Schauer, Structurally colored thiol chitosan thin films as a platform for aqueous heavy metal ion detection, Biomacromolecules 9 (2008) 289-295.

[40] S.K. Rastogi, P. Pal, D.E. Aston, T.E. Bitterwolf, A.L. Branen, 8-Aminoquinoline functionalized silica nanoparticles: a fluorescent nanoprobe for detection of divalent zinc in aqueous and in yeast cell suspension, ACS Appl. Mater. Interface 3 (2011) 1731-1739.

[41] Z. Liu, G. Li, Q. Ma, L. Liu, X. Su, A near-infrared turn-on fluorescent nanoprobe for zinc(II) based on CuInS 2 quantum dots modified with 8-aminoquinoline, Microchim. Acta 181 (2014) 1385-1391.

[42] Q. Jiang, Z. Guo, Y. Zhao, F. Wang, L. Mao, In vivo fluorescence sensing of the salicylate-induced change of zinc ion concentration in the auditory cortex of rat brain, Analyst 140 (2015) 197-203.

[43] H. Xu, R. Miao, Z. Fang, X. Zhong, Quantum dot-based "turn-on" fluorescent probe for detection of zinc and cadmium ions in aqueous media, Anal. Chim. Acta 687 (2011) 82-88.

[44] M.J. Ruedas-Rama, E.A. Hall, Azamacrocycle activated quantum dot for zinc ion detection, Anal. Chem. 80 (2008) 8260-8268.

[45] Y. Li, X. Hu, X. Zhang, H. Cao, Y. Huang, Unconventional application of gold nanoclusters/Zn-MOF composite for fluorescence turn-on sensitive detection of zinc ion, Anal. Chim. Acta 1024 (2018) 145-152.

Xueqing Gao received her BS degree from Department of Food and Biology Engineering, Wenjing Institute of Yantai University, in 2017. She is studying for her master degree in College of Chemistry and Chemical Engineering, Yantai University. Her current research interest is electrochemiluminescence and fluorescence sensor for detection of metal ions and small biomolecules.

Xuming Zhuang received his $\mathrm{PhD}$ in analytical chemistry from the Department of Chemistry of Shandong University, Jinan, in 2013. In the same year, he joined in College of Chemistry and Chemical Engineering, Yantai University, as a lecturer. His current research interest focuses on preparation of novel sensors and biosensors of metal ions and small biomolecules, and applications to in vivo analysis.

Chunyuan Tian obtained her Ph.D. degree in in analytical chemistry from Nanjing University in 2013, China. She is a lecturer in College of Chemistry and Chemical Engineering, Yantai University. Her research is focused on the preparation of new functional nanomaterial and electrochemiluminescence sensing application.
Huitao Liu received her $\mathrm{PhD}$ degree in analytical chemistry from Lanzhou University, Lanzhou, in 2000. After a year of postdoctoral experience at the Department of Chemistry, National Sun Yat-sen University, Kaohsiung, Taiwan, she joined as a professor, College of Chemistry and Chemical Engineering, Yantai University. Her research interests include the development of food and medicine analysis methods; preparation of novel functionalized nanomaterials and their application in analytical chemistry.

Wing-Fu Lai received his MSc degree in Materials Engineering and Nanotechnology from City University of Hong Kong, and earned his $\mathrm{PhD}$ in Chemistry from the University of Hong Kong. He joined the faculty of School of Pharmaceutical Sciences at Shenzhen University in 2016, and is also holding an adjunct assistant professorship in the Department of Applied Biology and Chemical Technology at the Hong Kong Polytechnic University. His research interests cover the design of molecular probes for bioimaging, synthesis of polymeric materials for gene delivery and controlled drug release, and the development of nanoparticulate systems for theranostic applications.

Zhenguang Wang received his PhD from the Department of Material Science of City University of Hong Kong, in 2017. Then, he joined in College of Chemistry and Environmental Science, Hebei University as an assistant professor. His current research interest focuses on synthesis of luminescent materials and applications for sensors and LEDs.

Xuming Yang received his Bachelor degree in Applied Chemistry in 2013 and Master degree in Physical Chemistry in 2016 from Central South University, China. He is now finishing his Ph.D. thesis under the supervision of Prof. Andrey L. Rogach at City University of Hong Kong. His research is focused on electrode materials for batteries, with a particular emphasis on electrochemical theories and operating mechanisms of batteries.

Lingxin Chen has been a professor at Yantai Institute of Coastal Zone Research, Chinese Academy of Sciences, since 2009. He obtained his Ph.D. in analytical chemistry at Dalian Institute of Chemical Physics, Chinese Academy of Sciences, in 2003. During 2004-2009, he worked at Department of Chemistry, Tsinghua University, and Department of Applied Chemistry, Hanyang University, respectively. His research interests include the studies of novel properties of materials such as functionalized nanoparticles \& functional probe molecules for developing nanoscale biochemical analysis methods and molecular imprinting-based sample pretreatment technology.

Andrey L. Rogach is a Chair Professor of Photonics Materials and the Founding Director of the Centre for Functional Photonics at City University of Hong Kong. He received his Diploma in Chemistry and PhD in Physical Chemistry from the Belarusian State University in Minsk, and worked as a staff scientist at the Institute of Physical Chemistry of the University of Hamburg (1995-2002), and at the Department of Physics of the LudwigMaximilians-University of Munich (2002-2009), Germany, where he completed his Habilitation in Experimental Physics. His research focuses on synthesis, assembly, and optical spectroscopy of nanomaterials, and their use for energy and optoelectronic applications. 